\title{
STABILITY OF TRAVELING CURVED FRONTS IN A CURVATURE FLOW WITH DRIVING FORCE*
}

\author{
HIROKAZU NINOMIYA ${ }^{\dagger}$ AND MASAHARU TANIGUCHI ${ }^{\ddagger}$
}

\begin{abstract}
This paper is concerned with asymptotical stability of traveling curved fronts to a mean curvature flow with a constant driving force term in the two-dimensional Euclidean space. Our first result shows that, if a suitable bounded perturbation is added to a traveling curved front, it does not recover its shape at any positive time. This fact implies that boundedness of given perturbations is not enough for asymptotical stability. Then we prove that, if a given bounded perturbation decays at infinity, the perturbed traveling curved fronts always recover their shapes as time goes on. This fact holds true for any large perturbation if it decays at infinity.
\end{abstract}

1. Introduction. Pattern formation is one of the most attractive fields in applied mathematics. Since traveling waves play important roles on the pattern formation, many researchers have studied them. Traveling waves in an one-dimensional media or planar traveling fronts in the plane seem to be mainly investigated so far. Fife [6] studied corner layers in the Allen-Cahn dynamics, which gives a first step to study traveling waves with more complicated shapes.

This paper is concerned with the asymptotical stability of traveling curved fronts for a curvature flow with a constant driving force term in $\mathbb{R}^{2}$. Let $D(t)$ be a domain (connected open set) in $\mathbb{R}^{2}$. Define $\Gamma(t)=\partial D(t)$. Let $\boldsymbol{\nu}$ be the normal vector on $\Gamma(t)$ pointing from $D(t)$ to $D(t)^{\mathbf{c}}$. The curvature $H$ is given by $H=-\operatorname{div} \boldsymbol{\nu}^{\prime}$. A pair of $(\Gamma, \nu)$ is often called an interface or a phase boundary. We study the traveling curved fronts of the following equation

$$
V=H+k \text {. }
$$

This equation appears in the several fields. For example, this equation describes the phase boundary in in the Belousov-Zhabotinsky reaction ([1], [2], [5] [8]), and also in the Allen-Cahn model in Chemistry ([6], [3], [11]). It also describes the motion of filamentary vortex of the Ginzburg-Landau model if it is confined in a plane ([5]).

In this paper we are interested in traveling waves with non-simple shapes, and we study the stability for the simplest one as a first step to study that of more complicated ones.

If $\Gamma(t)$ is represented by the graph $y=u(x, t)$ with $D(t)=\{(x, y) \mid y<u(x, t)\}$, (1.1) is rewritten as

$$
u_{t}=\frac{u_{x x}}{1+u_{x}^{2}}+k \sqrt{1+u_{x}^{2}}
$$

If the traveling front is represented by $u(x, t)=\varphi(x)+c t$ for the suitable coordinate where $c$ is a speed of the traveling front. Then $\varphi$ and $c$ satisfy

$$
c=\frac{\varphi_{x x}}{1+\varphi_{x}^{2}}+k \sqrt{1+\varphi_{x}^{2}}
$$

This equation (1.3) has a special solution called $V$-form waves or traveling curved fronts ([1], [2], [8], [9]).

\footnotetext{
* Dedicated to Professor Norio Shimakura on the occasion of his sixtieth birthday.

†Department of Mathematics and Informatics, Ryukoku University, Seta, Otsu 520-2194, Japan.

$\ddagger$ Department of Mathematical and Computing Sciences, Tokyo Institute of Technology, Tokyo 152-8552, Japan.
} 


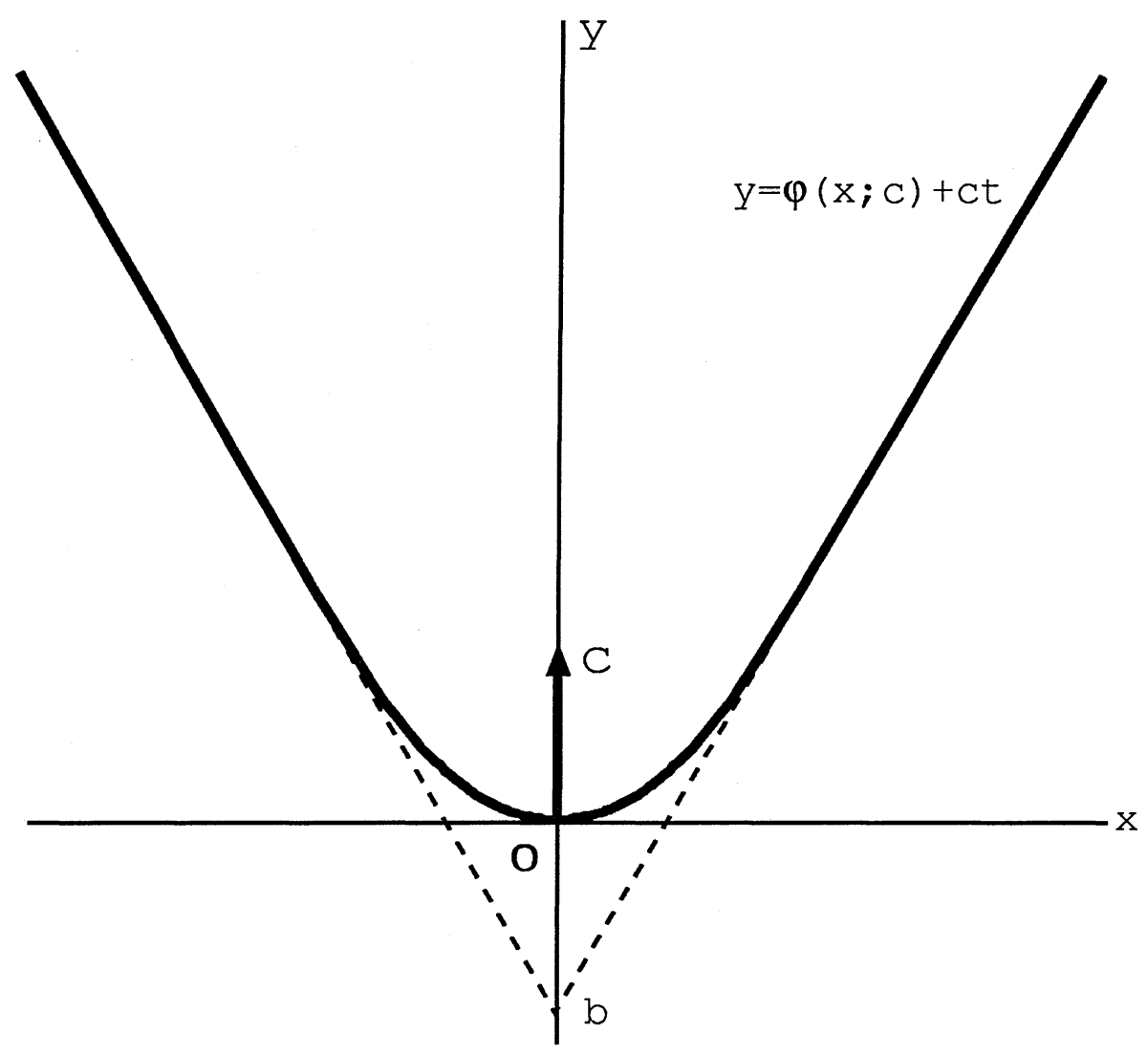

FIG. 1.1. The graph of a traveling curved front. The dotted lines represents $y=$ $\left(\sqrt{c^{2}-k^{2}} / k\right)|x|+b+c t$.

Proposition 1.1 ([9]) For $c>k>0$, there exists a solution $\varphi(x ; c)$ of (1.3) with $\varphi(0 ; c)=0, \varphi_{x}(0 ; c)=0$. The graph of $y=\varphi(x ; c)$ can be parametrized by $\theta=\arctan \varphi_{x}(x ; c)$ as

$$
\begin{aligned}
& x(\theta ; c):=\frac{\theta}{c}+\frac{k}{c \sqrt{c^{2}-k^{2}}} \log \left|\frac{1+\sqrt{\frac{c+k}{c-k}} \tan \frac{\theta}{2}}{1-\sqrt{\frac{c+k}{c-k}} \tan \frac{\theta}{2}}\right| \\
& y(\theta ; c):=-\frac{1}{c} \log \left(\frac{c \cos \theta-k}{c-k}\right),
\end{aligned}
$$

for $\theta \in\left(-\theta_{0}, \theta_{0}\right)$, where $\theta_{0}:=\arctan \left(\sqrt{c^{2}-k^{2}} / k\right) \in(0, \pi / 2)$ (see Fig. 1.1).

We denote the interface defined by $\varphi$ by

$$
\mathrm{TF}_{ \pm}\left(t ; c, x_{0}, y_{0}\right):=\left\{\left(x(\theta ;|c|)+x_{0}, \pm y(\theta ;|c|) \pm|c| t+y_{0}\right) \mid-\theta_{0}<\theta<\theta_{0}\right\}
$$

In [9, Theorem 1.2], all the traveling waves are classified into the following three types: lines, stationary circles and traveling curved fronts $\mathrm{TF}_{ \pm}\left(t ; c, x_{0}, y_{0}\right)$. This fact suggest that traveling curved fronts play an important role for (1.2). 
Hereafter we assume

$$
0<k<c
$$

We consider the stability of traveling curved fronts. We study (1.2) with an initial condition

$$
u(x, 0)=\varphi(x ; c)+\psi(x) .
$$

Here $\psi(x)$ is a given perturbation. We introduce

$$
B C^{1}:=\left\{\psi(x) \in C^{1}(\mathbb{R})\left|\sup _{x}\right| \psi(x)\left|<\infty, \sup _{x}\right| \psi_{x}(x) \mid<\infty\right\} .
$$

If the given perturbation is unbounded at infinity and change the asymptotic lines of $y=\varphi(x ; c)$, then the solution may converges to another traveling curved front $y=\varphi\left(x ; c^{\prime}\right)$ with a different speed. For bounded perturbations, it is easily seen that the traveling curved front $y=\varphi(x)+c t$ is stable in $L^{\infty}(\mathbb{R})$. So, we concentrate on the asymptotical stability for bounded perturbations. If $\psi(x) \in B C^{1}$, then the Cauchy problem (1.2)-(1.6) is well defined and has a unique classical solution up to $t=+\infty$. For the proof see [9]. See also [7] and [4]. Deckelnick, Elliott and Richardson [5] studied the stability problem in the half plane equipped with a boundary condition on the $y$-axis. Unfortunately only restricted perturbations are studied.

We should study more general perturbations including the ones with non-compact supports, which gives us a difficult problem, that is, what perturbations are admissible for asymptotical stability. This stability problem is not simple. In $\S 4$ we present an example where the given perturbation is bounded in $B C^{1}$ and the solution $u(x, t)$ does not converges to $\varphi(x ; c)+\alpha+c t$ for any $\alpha$. So we have to add another condition for $\psi(x)$. The following is the main assertion in this paper.

Theorem 1.1 For any given $\psi(x) \in B C^{1}$ with $\lim _{|x| \rightarrow \infty} \psi(x)=0$, the solution $u(x, t)$ of (1.2) with an initial data

$$
u(x, 0)=\varphi(x ; c)+\psi(x)
$$

converges to $\varphi(x ; c)+$ ct in $L^{\infty}(\mathbb{R})$ as $t \rightarrow \infty$. Namely, for any given $\varepsilon>0$, there exists a positive constant $T$ such that

$$
\sup _{-\infty<x<\infty}|u(x, t)-(\varphi(x ; c)+c t)|<\varepsilon
$$

holds true for all $t>T$.

That is, the traveling curved front $y=\varphi(x ; c)+c t$ is asymptotically stable for all $\psi(x) \in B C^{1}$ which decay at infinity. This implies the asymptotical stability of $y=\varphi(x ; c)+c t$ globally in space.

We also remark that if

$$
\lim _{x \rightarrow \pm \infty} \psi(x)=p_{ \pm}
$$

exist, then $u(x, t)$ converges to $\varphi\left(x-x_{0} ; c\right)+c t+y_{0}$, where

$$
x_{0}:=\frac{k\left(p_{-}-p_{+}\right)}{2 \sqrt{c^{2}-k^{2}}}, \quad y_{0}:=\frac{p_{+}+p_{-}}{2} .
$$


This paper is organized as follows. In $\S 2$ we study the asymptotical stability for traveling curved fronts locally in space. In $\S 3$ we prove the main theorem. In $\S 4$ we present an example where the given perturbation is bounded in $L^{\infty}(\mathbb{R})$, and the solution $u(x, t)$ does not converges to $\varphi(x ; c)+\alpha+c t$ for any $\alpha$.

2. Asymptotical stability locally in space. Let $y=\varphi(x ; c)$ be the traveling curved front as in Proposition 1.1. Hereafter we denote it simply by $y=\varphi(x)$. In this section we study the asymptotical stability of $y=\varphi(x)$ locally in space.

Define

$$
L[u]:=u_{t}-\frac{u_{x x}}{1+u_{x}^{2}}-\sqrt{1+u_{x}^{2}}
$$

We call $w_{+}(x, t)$ a supersolution if $L\left[w_{+}(x, t)\right] \geq 0$ for $x \in \mathbb{R}$ and $t>0$. A subsolution $w_{-}(x, t)$ can be defined similarly by using $L\left[w_{-}(x, t)\right] \leq 0$ instead of $L\left[w_{+}(x, t)\right] \geq 0$. We search for a supersolution and a subsolution of the following form

$$
w_{ \pm}(x, t)=\frac{1}{\alpha_{ \pm}(t)} \varphi\left(\alpha_{ \pm}(t) x\right)+c t+\beta_{ \pm}(t)
$$

with suitable $\alpha_{ \pm}(t)$ and $\beta_{ \pm}(t)$.

We begin with several lemmas.

LEMMA 2.1 The following properties hold true for $\varphi(x)$.

(i) The following asymptotic estimates

$$
\begin{aligned}
\varphi_{x}(x) & = \pm \frac{\sqrt{c^{2}-k^{2}}}{k}+O\left(e^{-c \sqrt{c^{2}-k^{2}}|x| / k}\right) \quad \text { as } x \rightarrow \pm \infty \\
\varphi(x) & =\frac{\sqrt{c^{2}-k^{2}}}{k}|x|+b+O\left(|x| e^{-c \sqrt{c^{2}-k^{2}}|x| / k}\right) \quad \text { as } x \rightarrow \pm \infty
\end{aligned}
$$

hold (see Fig. 1.1), where

$$
b:=-\frac{\sqrt{c^{2}-k^{2}}}{c k} \arctan \frac{\sqrt{c^{2}-k^{2}}}{k}-\frac{1}{c} \log \frac{2(c+k)}{c}<0 .
$$

(ii) A function $x \varphi_{x}(x)-\varphi(x)$ is strictly monotone increasing in $|x|$ with

$$
\begin{aligned}
0 \leq x \varphi_{x}(x)-\varphi(x) & <|b| \quad \text { for all } x \in \mathbb{R}, \\
\left.\left\{x \varphi_{x}(x)-\varphi(x)\right\}\right|_{x=0} & =0 \\
\lim _{|x| \rightarrow \infty}\left(x \varphi_{x}(x)-\varphi(x)\right) & =|b| .
\end{aligned}
$$

(iii) A function $c-k \sqrt{1+\varphi_{x}(x)^{2}}$ is strictly monotone decreasing in $|x|$ with

$$
\begin{aligned}
\left.\left\{c-k \sqrt{1+\varphi_{x}(x)^{2}}\right\}\right|_{x=0} & =c-k, \\
\lim _{|x| \rightarrow \infty}\left(c-k \sqrt{1+\varphi_{x}(x)^{2}}\right) & =0 .
\end{aligned}
$$

(iv) For $0<\alpha<1, \varphi(x)-\alpha^{-1} \varphi(\alpha x)$ is strictly monotone increasing in $|x|$ with

$$
0 \leq \varphi(x)-\frac{1}{\alpha} \varphi(\alpha x)<|b|\left(\frac{1}{\alpha}-1\right) \quad \text { for } 0<\alpha<1, x \in \mathbb{R} .
$$

For $\alpha>1, \alpha^{-1} \varphi(\alpha x)-\varphi(x)$ is strictly monotone increasing in $|x|$ with

$$
0 \leq \frac{1}{\alpha} \varphi(\alpha x)-\varphi(x)<|b|\left(1-\frac{1}{\alpha}\right) \quad \text { for } \alpha>1, x \in \mathbb{R} .
$$


Proof. The first statement (i) is a direct consequence from [9, Proposition 2.5]. For the second statement (ii) we use

$$
\left(x \varphi_{x}(x)-\varphi(x)\right)_{x}=x \varphi_{x x}(x),
$$

and

$$
\varphi_{x x}(x)>0 \quad \text { for } x \in \mathbb{R} .
$$

See [9] for this proof. Then we have

$$
\begin{array}{ll}
\left(x \varphi_{x}(x)-\varphi(x)\right)_{x}>0 & \text { if } x>0 \\
\left(x \varphi_{x}(x)-\varphi(x)\right)_{x}=0 & \text { if } x=0 \\
\left(x \varphi_{x}(x)-\varphi(x)\right)_{x}<0 & \text { if } x<0 .
\end{array}
$$

Using (i) we obtain (ii). Since $\left|\varphi_{x}(x)\right|$ is strictly monotone increasing in $|x|$, the statement (iii) follows. For $\alpha>0$, we have

$$
\begin{aligned}
\varphi(x)-\frac{1}{\alpha} \varphi(\alpha x) & =\int_{\alpha}^{1} \frac{\partial}{\partial \xi}\left(\frac{1}{\xi} \varphi(\xi x)\right) d \xi \\
& =\int_{\alpha}^{1} \frac{1}{\xi^{2}}\left(\xi x \varphi_{x}(\xi x)-\varphi(\xi x)\right) d \xi
\end{aligned}
$$

Then using (ii) we obtain (iv).

We search for a supersolution and a subsolution of the following type:

$$
w(x, t)=\frac{1}{\alpha(t)} \varphi(\alpha(t) x)+c t+\beta(t) .
$$

We set

$$
z=\alpha(t) x
$$

Then we have

$$
\begin{aligned}
L[w] & =w_{t}-\frac{w_{x x}}{1+w_{x}^{2}}-k \sqrt{1+w_{x}^{2}} \\
& =\frac{\alpha_{t}}{\alpha^{2}}\left(z \varphi_{z}(z)-\varphi(z)\right)+c+\beta_{t}-\frac{\alpha \varphi_{z z}(z)}{1+\varphi_{z}(z)^{2}}-k \sqrt{1+\varphi_{z}(z)^{2}} \\
& =\frac{\alpha_{t}}{\alpha^{2}}\left\{z \varphi_{z}(z)-\varphi(z)+\frac{(1-\alpha) \alpha^{2}}{\alpha_{t}}\left(c-k \sqrt{1+\varphi_{z}(z)^{2}}\right)+\frac{\beta_{t} \alpha^{2}}{\alpha_{t}}\right\} .
\end{aligned}
$$

We define $w_{ \pm}(x, t)$ as follows.

$$
\begin{aligned}
& w_{+}(x, t)=\frac{1}{\alpha_{+}(t)} \varphi\left(\alpha_{+}(t) x\right)+\beta_{+}(t)+c t, \\
& w_{-}(x, t)=\frac{1}{\alpha_{-}(t)} \varphi\left(\alpha_{-}(t) x\right)+\beta_{-}(t)+c t,
\end{aligned}
$$

where

$$
\begin{aligned}
& \alpha_{+}(t):=1-\delta e^{-\gamma t}, \\
& \beta_{+}(t):=\sigma\left(\frac{1}{\alpha_{+}(t)}-1\right)+\frac{(|b|-\sigma) \delta}{1-\delta}, \\
& \alpha_{-}(t):=1+\delta e^{-\gamma t}, \\
& \beta_{-}(t):=\sigma\left(\frac{1}{\alpha_{-}(t)}-1\right)-\frac{(|b|-\sigma) \delta}{1+\delta} .
\end{aligned}
$$


We also define

$$
\Sigma(\gamma, \delta):=\inf _{-\infty<z<\infty}\left\{z \varphi_{z}(z)-\varphi(z)+\frac{(1-\delta)^{2}}{\gamma}\left(c-k \sqrt{1+\varphi_{z}(z)^{2}}\right)\right\}
$$

By Lemma 2.1 (ii) and (iii), we have

$$
\begin{gathered}
0<\Sigma(\gamma, \delta) \leq|b| \\
\lim _{\gamma \rightarrow+0} \Sigma(\gamma, \delta)=|b| .
\end{gathered}
$$

Then we have the following assertion.

\section{LEMMA 2.2 If $\sigma, \gamma, \delta$ satisfy}

$$
\gamma>0, \quad 0<\sigma<\Sigma(\gamma, \delta), \quad 0<\delta<1
$$

then $w_{ \pm}(x, t)$ defined in (2.2) and (2.3) are a supersolution and a subsolution to (1.1), respectively. Moreover one has

$$
w_{-}(x, 0)<\varphi(x)<w_{+}(x, 0) \quad \text { for all } x \in \mathbb{R},
$$

and

$$
\lim _{|x| \rightarrow \infty}\left|w_{ \pm}(x, 0)-\varphi(x)\right|=0
$$

Proof. Substituting $\alpha_{ \pm}(t)$ and $\beta_{ \pm}(t)$ into (2.1) yields

$$
L\left[w_{ \pm}\right]= \pm \frac{\gamma \delta e^{-\gamma t}}{\alpha_{ \pm}(t)^{2}}\left\{z \varphi_{z}(z)-\varphi(z)+\frac{\alpha_{ \pm}(t)^{2}}{\gamma}\left(c-k \sqrt{1+\varphi_{z}(z)^{2}}\right)-\sigma\right\} .
$$

By Lemma 2.1 (ii) and (iii), $\Sigma(\gamma, \delta)>0$. If $0<\sigma<\Sigma(\gamma, \delta)$,

$$
z \varphi_{z}(z)-\varphi(z)+\frac{\alpha_{ \pm}(t)^{2}}{\gamma}\left(c-k \sqrt{1+\varphi_{z}(z)^{2}}\right)-\sigma \geq \Sigma(\gamma, \delta)-\sigma>0 .
$$

Thus $w_{ \pm}$is a supersolution and a subsolution, respectively.

Next we study for $w_{+}(x, 0)-\varphi(x)$. We have

$$
w_{+}(x, 0)-\varphi(x)=\frac{1}{1-\delta} \varphi((1-\delta) x)-\varphi(x)+\frac{|b| \delta}{1-\delta} .
$$

By Lemma 2.1 (iv),

$$
w_{+}(x, 0)-\varphi(x)>0
$$

follows. Similarly, we have

$$
w_{-}(x, 0)-\varphi(x)=\frac{1}{1+\delta} \varphi((1+\delta) x)-\varphi(x)-\frac{|b| \delta}{1+\delta}<0 .
$$

The estimate $\lim _{|x| \rightarrow \infty}\left|w_{ \pm}(x, 0)-\varphi(x)\right|=0$ is a direct consequence of Lemma 2.1. 口 
By Lemma 2.1 (iv),

$$
\begin{aligned}
& 0 \leq w_{+}(x, t)-\varphi(x, t)-c t \leq \frac{\delta \sigma e^{-\gamma t}}{1-\delta e^{-\gamma t}}+\frac{(|b|-\sigma) \delta}{1-\delta} \\
& 0 \leq \varphi(x, t)+c t-w_{-}(x, t) \leq \frac{\delta \sigma e^{-\gamma t}}{1+\delta e^{-\gamma t}}+\frac{(|b|-\sigma) \delta}{1+\delta}
\end{aligned}
$$

Then we have the following theorem.

TheOREM 2.1 (ASYMPTOTICAL STABILITY LOCALly IN SPACE) Let $\varepsilon>0$ be arbitrarily given. If $\delta, \gamma, \sigma$ satisfy

$$
0<\delta<\frac{\varepsilon}{2|b|-2 \sigma+\varepsilon}, \quad 0<\sigma<\Sigma(\gamma, \delta), \quad \gamma>0
$$

and $u(x, 0) \in B C^{1}$ satisfies

$$
w_{-}(x, 0) \leq u(x, 0) \leq w_{+}(x, 0) \quad x \in \mathbb{R},
$$

then there exists $T_{0}>0$ such that

$$
\sup _{x}|u(x, t)-\varphi(x)-c t|<\varepsilon
$$

for $t>T_{0}$.

Proof. From the assumption on $\delta, \gamma, \sigma$, we have

$$
\max \left\{\frac{(|b|-\sigma) \delta}{1-\delta}, \frac{(|b|-\sigma) \delta}{1+\delta}\right\}<\frac{\varepsilon}{2}
$$

Then Theorem 2.1 follows from Lemma 2.2 and the the Phragmèn-Lindelöf maximum principle [10, Theorem 10, Chap. 3].

Thus the traveling front $\mathrm{TF}_{ \pm}\left(t ; c, x_{0}, y_{0}\right)$ is asymptotically stable locally in space.

3. Asymptotical stability globally in space. In this section, we study the stability of traveling curved fronts for more general initial condition, and prove Theorem 1.1. Define

$$
B C_{0}^{1}:=\left\{\psi(x) \in C^{1}(\mathbb{R})\left|\sup _{x}\right| \psi(x)\left|<\infty, \sup _{x}\right| \psi_{x}(x) \mid<\infty, \lim _{x \rightarrow \infty} \psi(x)=0\right\} .
$$

LEMMA 3.1 For any $\varepsilon>0$ and any $\psi(x) \in B C_{0}^{1}$, there exists a constant $\delta_{1} \in(0,1)$ such that

$$
\varphi(x)+\psi(x)<\frac{1}{1-\delta} \varphi((1-\delta) x)+\frac{|b| \delta}{1-\delta}+\varepsilon \quad \text { for all } x \in \mathbb{R} \text { and } 0<\delta \leq \delta_{1} .
$$

Proof. From the assumption on $\psi(x)$, there exists sufficiently large $R_{1}>0$ with

$$
|\psi(x)|<\varepsilon \quad \text { for all }|x|>R_{1} .
$$


We have

$$
\frac{1}{1-\delta} \varphi((1-\delta) x)+\frac{|b| \delta}{1-\delta}-\varphi(x)>0 \quad \text { for all } x \in \mathbb{R}
$$

from Lemma 2.1. Thus we get

$$
\varphi(x)+\psi(x)<\frac{1}{1-\delta} \varphi((1-\delta) x)+\frac{|b| \delta}{1-\delta}+\varepsilon \quad \text { for }|x|>R_{1} .
$$

For $|x| \leq R_{1}$, we have

$$
\begin{aligned}
& \frac{1}{1-\delta} \varphi((1-\delta) x)+\frac{|b| \delta}{1-\delta}-\varphi(x)-\psi(x) \\
\geq & \frac{1}{1-\delta} \varphi\left((1-\delta) R_{1}\right)+\frac{|b| \delta}{1-\delta}-\varphi\left(R_{1}\right)-\max _{|x| \leq R_{1}}|\psi(x)|
\end{aligned}
$$

by Lemma 2.1 (iv). The right-hand side goes to $+\infty$ as $\delta \rightarrow 1-0$ by virtue of

$$
\lim _{\delta \rightarrow 1-0} \frac{1}{1-\delta} \varphi\left((1-\delta) R_{1}\right)=0 .
$$

Thus we can choose $\delta_{1} \in(0,1)$ sufficiently close to 1 such that

$$
\varphi(x)+\psi(x)<\frac{1}{1-\delta} \varphi((1-\delta) x)+\frac{|b| \delta}{1-\delta} \quad \text { for }|x| \leq R_{1} \text { and } \delta \in\left(0, \delta_{1}\right) .
$$

Combining the two inequalities stated above, we complete the proof.

For any given $\varepsilon>0$ and $\psi(x) \in B C_{0}^{1}, \delta_{+}, \sigma_{+}$and $\gamma_{+}$are chosen such that

$$
0<\delta_{+}<\min \left\{\frac{\varepsilon}{2|b|+\varepsilon}, \delta_{1}\right\}, \quad 0<\sigma_{+}<\Sigma\left(\gamma_{+}, \delta_{+}\right), \quad \gamma_{+}>0
$$

hold true. Let $w_{+}(x, t)$ be defined as in $(2.2)$ with $(\delta, \gamma, \sigma)=\left(\delta_{+}, \gamma_{+}, \sigma_{+}\right)$. By Lemmas 2.2 and $3.1, w_{+}(x, t)$ is a supersolution with $u(x, 0)<w_{+}(x, 0)$. Then by the comparison principle, we have

$$
u(x, t)<w_{+}(x, t)
$$

where $u(x, t)$ is the solution of $(1.2)$ with $u(x, 0)=\varphi(x)+\psi(x)$. By (2.6), we see that there exists $T>0$ satisfying

$$
u(x, t) \leq \varphi(x)+c t+2 \varepsilon \quad \text { for } x \in \mathbb{R}, t>T .
$$

This gives an upper estimate for $u(x, t)$.

Next we consider a subsolution, and search for a lower estimate to prove the global attractivity of the traveling curved front. We cannot obtain a lower estimate by a parallel way to get (3.2) unfortunately. We need the help of another subsolution, which contacts the $y$-axis tangentially. For this purpose, we use the following another traveling curved front.

LEMMA 3.2 ([9]) For $c>k>0$, (1.3) has a unique solution $\varphi^{*}(x ; c)$ that is tangential to the $y$-axis in the half plane $\{x>0\}$ with $\varphi^{*}(0 ; c)=0$. More exactly, the 


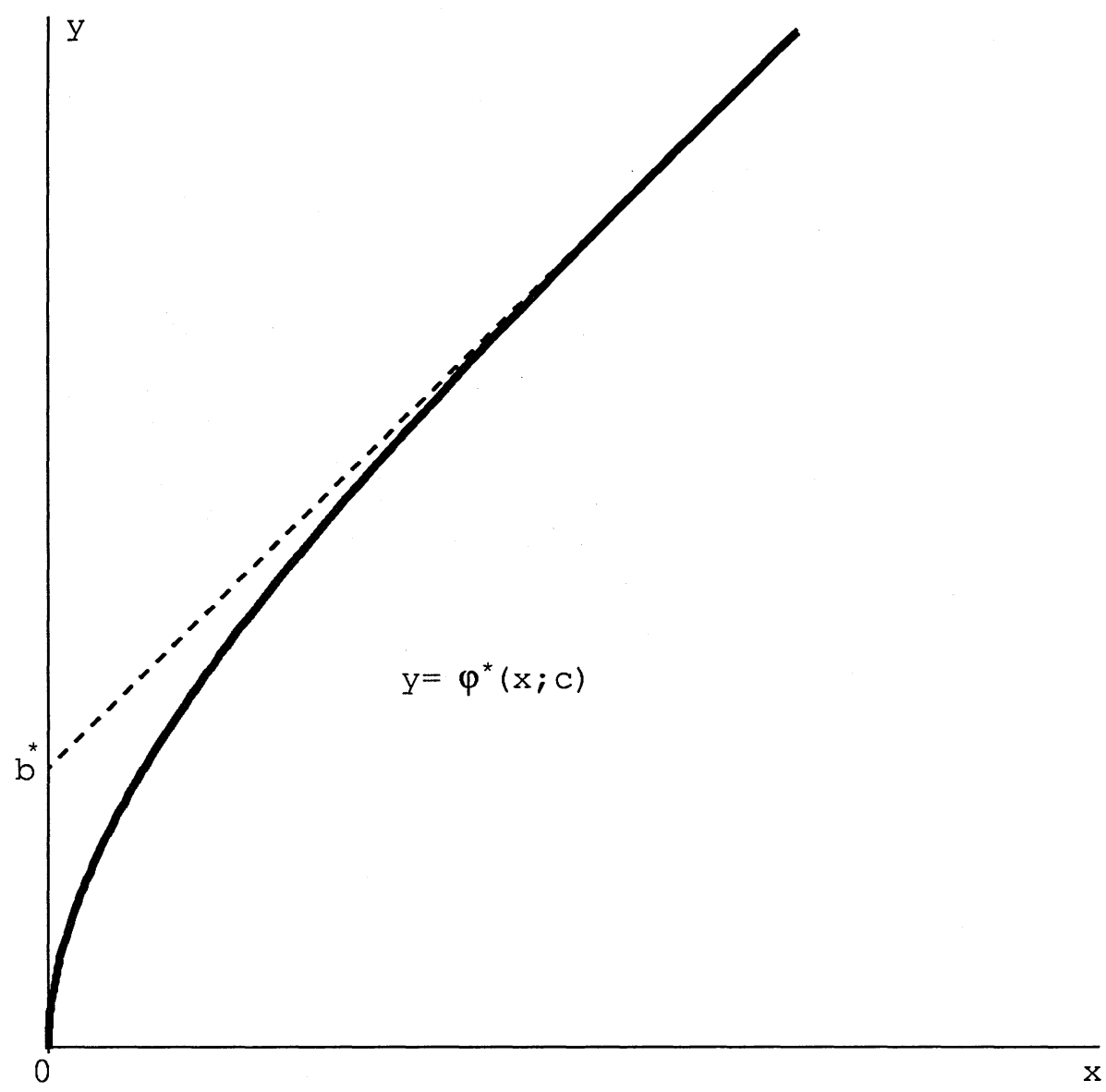

FIG. 3.1. The graph of $y=\varphi^{*}(x ; c)$. The dotted line represents $y=\left(\sqrt{c^{2}-k^{2}} / k\right) x+b^{*}$.

graph $y=\varphi^{*}(x ; c)$ is parametrized in terms of $\theta=\arctan \varphi_{x}^{*}(x ; c) \in\left(\theta_{0}, \pi / 2\right)$ as

$$
x^{*}(\theta):=\frac{2 \theta-\pi}{2 c}+\frac{k}{c \sqrt{c^{2}-k^{2}}} \log \frac{\left(\sqrt{\frac{c+k}{c-k}} \tan \frac{\theta}{2}+1\right)\left(\sqrt{\frac{c+k}{c-k}}-1\right)}{\left(\sqrt{\frac{c+k}{c-k}} \tan \frac{\theta}{2}-1\right)\left(\sqrt{\frac{c+k}{c-k}}+1\right)}
$$

(3.4) $y^{*}(\theta):=-\frac{1}{c} \log \frac{k-c \cos \theta}{k}$.

Here $\theta_{0}$ is as in Proposition 1.1.

We denote $\varphi^{*}(|x| ; c)$ simply by $\varphi^{*}(x)$. Then it is an even function defined for $x \in \mathbb{R}$. The function $\varphi^{*}(x)$ possesses the following properties (see Fig. 3.1).

LEMMA 3.3 The following properties hold true for $\varphi^{*}(x)$.

(i) $\varphi^{*}(x)$ satisfies

$$
\lim _{x \rightarrow \pm 0} \sqrt{|x|} \varphi_{x}^{*}(x)= \pm \sqrt{2 k}
$$


and also

$$
\begin{aligned}
& \varphi_{x}^{*}(x)= \pm \frac{\sqrt{c^{2}-k^{2}}}{k}+O\left(e^{-c \sqrt{c^{2}-k^{2}}|x| / k}\right) \quad \text { as } x \rightarrow \pm \infty, \\
& \varphi^{*}(x)=\frac{\sqrt{c^{2}-k^{2}}}{k}|x|+O\left(|x| e^{-c \sqrt{c^{2}-k^{2}}|x| / k}\right) \quad \text { as } x \rightarrow \pm \infty \text {. }
\end{aligned}
$$

Here

$$
b^{*}:=\frac{\sqrt{c^{2}-k^{2}}}{c k}\left(\frac{\pi}{2}-\arctan \frac{\sqrt{c^{2}-k^{2}}}{k}\right)+\frac{1}{c} \log \frac{c\left(c+\sqrt{c^{2}-k^{2}}\right)}{2\left(c^{2}-k^{2}\right)}>0 .
$$

(ii) A function $\varphi^{*}(x)-x \varphi_{x}^{*}(x)$ is strictly monotone increasing function in $|x|$ with

$$
\begin{aligned}
& 0<\varphi^{*}(x)-x \varphi_{x}^{*}(x)<b^{*} \quad \text { for } x \in(-\infty, 0) \cup(0, \infty), \\
& \lim _{x \rightarrow 0}\left(\varphi^{*}(x)-x \varphi_{x}^{*}(x)\right)=0 \\
& \lim _{|x| \rightarrow \infty}\left(\varphi^{*}-x \varphi_{x}^{*}(x)\right)=b^{*}
\end{aligned}
$$

(iii) A function $k \sqrt{1+\varphi_{x}^{*}(x)^{2}}-c$ is strictly monotone decreasing in $|x|$ with

$$
\begin{aligned}
& \lim _{|x| \rightarrow 0}\left(k \sqrt{1+\varphi_{x}^{*}(x)^{2}}-c\right)=+\infty, \\
& \lim _{|x| \rightarrow \infty}\left(k \sqrt{1+\varphi_{x}^{*}(x)^{2}}-c\right)=0 .
\end{aligned}
$$

(iv) For $x \in \mathbb{R}$ and $\delta \in(0,1)$, let $F(x, \delta)$ be defined by

$$
F(x, \delta):=\varphi(x)-\frac{1}{1-\delta} \varphi^{*}((1-\delta) x)+\frac{b^{*}}{1-\delta}+|b| .
$$

Then $F(x, \delta)$ is strictly decreasing in $|x|$, and is strictly increasing in $\delta \in$ $(0,1)$. It satisfies

$$
0<F(x, \delta) \leq \frac{b^{*}}{1-\delta}+|b| \quad \text { for } x \in \mathbb{R}, 0<\delta<1
$$

and

$$
\lim _{\delta \rightarrow 1-0} \min _{|x| \leq R_{2}} F(x, \delta)=+\infty \quad \text { for any fixed } R_{2}>0 .
$$

(i) For $x \in \mathbb{R}, \alpha \in(0,1]$ and $\sigma \in\left(0, b^{*}\right)$, let $G(x, \alpha)$ be defined by

$$
G(x, \alpha)=\frac{1}{\alpha} \varphi^{*}(\alpha x)-\frac{\sigma}{\alpha} .
$$

Then it satisfies

$$
-\left(b^{*}-\sigma\right)\left(\frac{1}{\alpha_{1}}-\frac{1}{\alpha_{2}}\right)<G\left(x, \alpha_{2}\right)-G\left(x, \alpha_{1}\right) \leq \sigma\left(\frac{1}{\alpha_{1}}-\frac{1}{\alpha_{2}}\right)
$$

for $0<\alpha_{1}<\alpha_{2} \leq 1$. 
Proof. We can easily show (i), (ii), (iii) by Proposition 2.5 in [9] and the argument similar to the proof of Lemma 2.1. For (iv), it suffices to show it for $x \geq 0$, because $F(x, \delta)$ is an even function in $x$. We have

$$
\frac{\partial}{\partial \delta} F(x, \delta)=\frac{1}{(1-\delta)^{2}}\left\{(1-\delta) x \varphi_{x}^{*}((1-\delta) x)-\varphi^{*}((1-\delta) x)+b^{*}\right\}>0
$$

for all $x \geq 0$ and $0<\delta<1$. By (ii), $F(x, \delta)$ is strictly increasing in $\delta$. Using

$$
\begin{aligned}
\varphi_{x x}^{*}(x)>0 & \text { for } x>0 \\
\varphi_{x}(x)<\frac{\sqrt{c^{2}-k^{2}}}{k}<\varphi_{x}^{*}(x) & \text { for } x>0
\end{aligned}
$$

we have

$$
\begin{aligned}
F_{x x}(x, \delta) & =\varphi_{x x}(x)-(1-\delta) \varphi_{x x}^{*}((1-\delta) x)>0 \quad \text { for } x>0 \\
F_{x}(x, \delta) & =\varphi_{x}(x)-\varphi_{x}^{*}((1-\delta) x)<0 \quad \text { for } x>0
\end{aligned}
$$

Thus $F(x, \delta)$ is strictly convex in $x>0$, and since it satisfies

$$
\begin{array}{r}
F(0, \delta)=\frac{b^{*}}{1-\delta}+|b|>0 \\
\lim _{|x| \rightarrow+\infty} F(x, \delta)=0
\end{array}
$$

we obtain

$$
0<F(x, \delta)<\frac{b^{*}}{1-\delta}+|b| \text { for all } x>0 \text { and } 0<\delta<1
$$

Since

$$
\begin{aligned}
\frac{\partial}{\partial \delta} F(x, \delta) & =\frac{1}{(1-\delta)^{2}}\left\{(1-\delta) x \varphi_{x}^{*}((1-\delta) x)-\varphi^{*}((1-\delta) x)+b^{*}\right\} \\
& \geq \frac{1}{(1-\delta)^{2}}\left\{R_{2} \varphi_{x}^{*}\left(R_{2}\right)-\varphi^{*}\left(R_{2}\right)+b^{*}\right\}>0
\end{aligned}
$$

for $|x| \leq R_{2}$, we have

$$
F(x, \delta) \geq F(x, 0)+\frac{\delta}{1-\delta}\left\{R_{2} \varphi_{x}^{*}\left(R_{2}\right)-\varphi^{*}\left(R_{2}\right)+b^{*}\right\} \quad \text { for }|x| \leq R_{2} .
$$

This yields (3.5), which completes the proof of (iv).

For (v), we have

$$
\frac{\partial}{\partial \alpha} G(x, \alpha)=-\frac{1}{\alpha^{2}}\left(\varphi^{*}(\alpha x)-\alpha x \varphi_{x}^{*}(\alpha x)-\sigma\right) .
$$

Integrating over $\left(\alpha_{1}, \alpha_{2}\right)$, we get

$$
G\left(x, \alpha_{2}\right)-G\left(x, \alpha_{1}\right)=-\int_{\alpha_{1}}^{\alpha_{2}} \frac{1}{\alpha^{2}}\left(\varphi^{*}(\alpha x)-\alpha x \varphi_{x}^{*}(\alpha x)-\sigma\right) d \alpha .
$$

Combining this equality and (ii), we obtain (v). 
Since $\varphi^{*}(x)$ has a singularity in $x=0$, the following maximum principle needs a proof.

Proposition 3.4 Let $u$ be continuously differentiable in $t>0$ and twice continuously differentiable in $x \in \mathbb{R}$ satisfying

$$
L[u] \geq 0, \quad \text { for } t>0, x \in(-\infty, \infty),
$$

and let $v$ be continuously differentiable in $t>0$ and twice continuously differentiable in $x \neq 0$ satisfying

$$
L[v] \leq 0, \text { for } t>0, x \in(-\infty, 0) \cup(0, \infty) .
$$

Assume

$$
u(x, 0) \geq v(x, 0) \text { in } x \in \mathbb{R}
$$

and

$$
\sup _{t>0,-\infty<x<\infty}\left|u_{x}(x, t)\right|<+\infty
$$

and for any $\eta>0$,

$$
\sup _{t>0,|x| \geq \eta}\left|v_{x}(x, t)\right| \leq M_{\eta}, \text { and } \lim _{x \rightarrow+0} v_{x}(x, t)=\infty, \lim _{x \rightarrow-0} v_{x}(x, t)=-\infty
$$

with some $M_{\eta} \in(0,+\infty)$. Then one has

$$
u(x, t) \geq v(x, t) \quad \text { for } t>0 \text { and } x \in \mathbb{R} .
$$

Proof. We prove by contradiction. Assume the contrary. Then there exist constants $T>0$ and $x_{0} \in \mathbb{R}$ with $v\left(x_{0}, T\right)>u\left(x_{0}, T\right)$. Define

$$
\tau=\inf \{t>0 \mid v(\xi, t)>u(\xi, t) \text { for some } \xi \in \mathbb{R}\} .
$$

The continuity of $u$ and $v$ implies that there exists $\tau^{\prime}$ such that

$$
v\left(\xi_{t}, t\right)>u\left(\xi_{t}, t\right) \text { for any } t \in\left(\tau, \tau^{\prime}\right) \text { and some } \xi_{t} \in \mathbb{R} .
$$

Assume that there exist $t_{n} \in\left(\tau, \tau^{\prime}\right)$ and $x_{n} \in \mathbb{R}$ such that

$$
v\left(x_{n}, t_{n}\right) \geq u\left(x_{n}, t_{n}\right), \quad \lim _{n \rightarrow \infty} t_{n}=\tau, \quad \lim _{n \rightarrow \infty} x_{n}=0 .
$$

Then we have

$$
v(0, \tau)=\lim _{n \rightarrow \infty} v\left(x_{n}, t_{n}\right) \geq \lim _{n \rightarrow \infty} u\left(x_{n}, t_{n}\right)=u(0, \tau) .
$$

By the definition of $\tau$, we have

$$
v(0, t) \leq u(0, t) \text { for } t<\tau
$$


Then $v(0, \tau)=u(0, \tau)$ follows. Using this equality, (3.6) and (3.7), we have

$$
v(x, \tau)>u(x, \tau) \text { for } x \neq 0 \text { and }|x| \text { small enough. }
$$

This contradicts the definition of $\tau$. Thus such $x_{n}$ and $t_{n}$ do not exist. This implies that there exists a positive constant $\xi_{0}$ satisfying

$$
v(x, t) \leq u(x, t) \text { for }|x| \leq \xi_{0} \text { and } t \in\left[\tau, \tau^{\prime}\right] .
$$

We can apply the Phragmèn-Lindelöf maximum principle [10, Theorem 10, Chap. 3] to $u-v$ in $\left(-\infty,-\xi_{0}\right] \cup\left[\xi_{0}, \infty\right) \times\left[\tau, \tau^{\prime}\right]$. Then we obtain

$$
v(x, t) \leq u(x, t) \quad \text { for }|x| \geq \xi_{0}, \tau \leq t \leq \tau^{\prime} .
$$

Combining (3.8) and (3.9), we have

$$
v(x, t) \leq u(x, t) \quad \text { for } x \in \mathbb{R}, \tau \leq t \leq \tau^{\prime} .
$$

This contradict the definition of $\tau$. This completes the proof.

Note that $u(x, t)$ given by (1.2)-(1.6) satisfies the assumption in Proposition 3.4 by Theorem 1.4 in [9]. Now we will construct another subsolution as

$$
w^{*}(x, t)=\frac{1}{\alpha(t)} \varphi^{*}(\alpha(t) x)-\frac{\sigma}{\alpha(t)}-\frac{b^{*}-\sigma}{1-\delta}-|b|+c t .
$$

Here we put

$$
\alpha(t)=1-\delta e^{-\gamma t}
$$

with $\delta \in(0,1), \gamma>0$ and $\sigma \in\left(0, b^{*}\right)$. We choose them with $0<\sigma<\Sigma^{*}(\gamma, \delta)$, where

$$
\Sigma^{*}(\gamma, \delta):=\inf _{x>0}\left\{\varphi^{*}(x)-x \varphi_{x}^{*}(x)+\frac{(1-\delta)^{2}}{\gamma}\left(k \sqrt{1+\varphi_{x}^{*}(x)^{2}}-c\right)\right\} .
$$

LEMMA 3.5 If

$$
0<\delta<1, \quad \gamma>0, \quad 0<\sigma<\Sigma^{*}(\gamma, \delta)
$$

then $w^{*}(x, t)$ satisfies

$$
L\left[w^{*}\right] \leq 0 \quad \text { for all } x \in \mathbb{R}, t>0,
$$

and thus it is a subsolution. For any $\varepsilon>0$ and any function $\psi(x) \in B C_{0}^{1}$, there exists a trio $\left(\delta^{*}, \gamma^{*}, \sigma^{*}\right)$ with (3.11) and

$$
\begin{aligned}
\frac{1}{1-\delta^{*}} \varphi^{*}\left(\left(1-\delta^{*}\right) x\right)-\frac{b^{*}}{1-\delta^{*}}-|b|-\frac{\varepsilon}{4} & <\varphi(x)+\psi(x) \quad \text { for all } x \in \mathbb{R}, \\
0 & <\frac{b^{*}-\sigma^{*}}{1-\delta^{*}}<\frac{\varepsilon}{4}
\end{aligned}
$$


Proof. Set $z=\alpha(t) z$. Then, by (2.1) we have

$$
L\left[w^{*}\right]=-\frac{\delta \gamma e^{-\gamma t}}{\alpha(t)^{2}}\left\{\varphi^{*}(z)-z \varphi_{z}^{*}(z)+\frac{\alpha(t)^{2}}{\gamma}\left(k \sqrt{1+\varphi_{z}^{*}(z)^{2}}-c\right)-\sigma\right\} .
$$

Hence we get

$$
L\left[w^{*}\right] \leq-\frac{\delta \gamma e^{-\gamma t}}{\alpha(t)^{2}}\left(\Sigma^{*}(\gamma, \delta)-\sigma\right)<0 .
$$

This implies that $w^{*}(x, t)$ is a subsolution.

From $\lim _{|x| \rightarrow \infty} \psi(x)=0$, there exists $R_{3}>0$ with

$$
-\frac{\varepsilon}{4}<\psi(x) \quad \text { for }|x|>R_{3} .
$$

Thus (3.12) follows from Lemma 3.3 (iv) for $|x|>R_{3}$. For $|x| \leq R_{3}$, we have

$$
\varphi(x)+\psi(x)-\frac{1}{1-\delta^{*}} \varphi^{*}\left(\left(1-\delta^{*}\right) x\right)+\frac{b^{*}}{1-\delta^{*}}+|b|+\frac{\varepsilon}{4} \geq F\left(x, \delta^{*}\right)-\max _{|x| \leq R_{3}}|\psi(x)|+\frac{\varepsilon}{4},
$$

where $F(x, \delta)$ is as in Lemma 3.3. By (3.5), we have (3.12) for $|x| \leq R_{3}$ if $\delta^{*}>0$ is close to 1 . Then we choose $\sigma^{*} \in\left(0, b^{*}\right)$ so close to $b^{*}$ that (3.13) is valid. Finally we choose $\gamma^{*}>0$ so small that it satisfies $\Sigma^{*}\left(\gamma^{*}, \delta^{*}\right)>\sigma^{*}$. We complete the proof.

We fix a subsolution $w^{*}(x, t)$ as in $(3.10)$ associated with a trio $\left(\delta^{*}, \sigma^{*}, \gamma^{*}\right)$ which satisfies (3.11), (3.12) and (3.13) in Lemma 3.5.

Proof of Theorem 1.1. From Proposition 3.4 and Lemma 3.5, we have

$$
w^{*}(x, t)-\frac{\varepsilon}{4} \leq u(x, t) \quad \text { for } x \in \mathbb{R}, t>0,
$$

which is equivalent to

$$
\frac{1}{\alpha^{*}(t)} \varphi^{*}\left(\alpha^{*}(t) x\right)-\frac{\sigma^{*}}{\alpha^{*}(t)}-\frac{b^{*}-\sigma^{*}}{1-\delta^{*}}-|b|+c t-\frac{\varepsilon}{4} \leq u(x, t)
$$

for $x \in \mathbb{R}$ and $t>0$ where

$$
\alpha^{*}(t):=1-\delta^{*} e^{-\gamma^{*} t}
$$

From Lemma $3.3(\mathrm{v})$, we have

$$
\begin{aligned}
\left(b^{*}-\sigma^{*}\right)\left(\frac{1}{\alpha^{*}(t)}-\frac{1}{1-\delta^{*}}\right)<\frac{1}{\alpha^{*}(t)} \varphi^{*}\left(\alpha^{*}(t) x\right) & \\
& -\frac{\sigma^{*}}{\alpha^{*}(t)}-\left(\frac{1}{1-\delta^{*}} \varphi^{*}\left(\left(1-\delta^{*}\right) x\right)-\frac{\sigma^{*}}{1-\delta^{*}}\right) .
\end{aligned}
$$

Combining these two inequalities, we obtain

$$
\begin{aligned}
\left(b^{*}-\sigma^{*}\right)\left(\frac{1}{\alpha^{*}(t)}-\frac{1}{1-\delta^{*}}\right)+ & \left(\frac{1}{1-\delta^{*}} \varphi^{*}\left(\left(1-\delta^{*}\right) x\right)-\frac{\sigma^{*}}{1-\delta^{*}}\right) \\
& -\frac{b^{*}-\sigma^{*}}{1-\delta^{*}}-|b|+c t-\frac{\varepsilon}{4} \leq u(x, t)
\end{aligned}
$$


From (3.13), we have

$$
-\frac{\varepsilon}{2}+\left(\frac{1}{1-\delta^{*}} \varphi^{*}\left(\left(1-\delta^{*}\right) x\right)-\frac{\sigma^{*}}{1-\delta^{*}}\right)-\frac{b^{*}-\sigma^{*}}{1-\delta^{*}}-|b|+c t \leq u(x, t) .
$$

From this inequality and Lemma 3.3 (i) and the fact $b<0$, there exists $R^{*}>0$ that is large enough and independent of $t$ with

$$
\frac{\sqrt{c^{2}-k^{2}}}{k} x+b+c t-\varepsilon \leq u(x, t)
$$

for $|x|>R^{*}, t>0$. Taking $R_{4}>R^{*}$ that is independent of $t>0$ and is large enough, we have

$$
\varphi(x)+c t-2 \varepsilon \leq u(x, t) \quad \text { for all }|x| \geq R_{4}, t>0 .
$$

Taking $R_{4}>0$ larger if necessary we can assume,

$$
|\psi(x)|<\varepsilon \quad \text { for all }|x| \geq R_{4} .
$$

Our aim is to show that there exists $T>0$ satisfying

$$
\varphi(x)+c t-2 \varepsilon \leq u(x, t) \quad \text { for all }|x|<R_{4}, t>T .
$$

Put $M=-\min _{|x| \leq R_{4}}\{\psi(x)\}$. If $M \leq 2 \varepsilon$, we have

$$
\varphi(x)-2 \varepsilon \leq \varphi(x)+\psi(x)=u(x, 0),
$$

and also

$$
\varphi(x)+c t-2 \varepsilon \leq u(x, t),
$$

which together with (3.2) gives the proof.

From now on we assume $M>2 \varepsilon$. Choose $\gamma=\gamma_{-}$with

$$
\Sigma\left(\gamma, \frac{1}{3}\right)>|b|-\frac{\varepsilon}{2}
$$

Set

$$
\sigma_{-}:=|b|-\frac{\varepsilon}{2}, \quad \delta_{-}:=\frac{1}{3} .
$$

We fix $(\sigma, \gamma, \delta)=\left(\sigma_{-}, \gamma_{-}, \delta_{-}\right)$, which enjoys (2.8). We also define $w_{-}(x, t)$ by $(2.3)$ with $(\sigma, \gamma, \delta)=\left(\sigma_{-}, \gamma_{-}, \delta_{-}\right)$. Define

$$
m:=\frac{|b| \delta_{-}}{1+\delta_{-}}=\frac{|b|}{4}=\sup _{-\infty<x<\infty}\left(\varphi(x)-w_{-}(x, 0)\right) .
$$

There exists sufficiently large $T_{0}>0$ with

$$
\sup _{-\infty<x<\infty}\left(\varphi(x)+c t-w_{-}(x, t)\right)<\varepsilon \quad \text { for all } t \geq T_{0}
$$

by virtue of Theorem 2.1 . 
We start with

$$
-M \leq\left.(u(x, t)-\varphi(x)-c t)\right|_{t=0} \quad \text { for }|x| \leq R_{4},
$$

which combined with $-2 \varepsilon \leq-M$ and (3.14) gives

$$
\varphi(x)-M \leq u(x, 0) \quad \text { for } x \in \mathbb{R} .
$$

Because $w_{-}(x, t)-M$ is a subsolution with

$$
w_{-}(x, 0)-M \leq \varphi(x)-M \leq u(x, 0)
$$

we obtain

$$
\varphi(x)+c t-M+(m-\varepsilon) \leq u(x, t) \quad \text { for } x \in \mathbb{R}, t \geq T_{0} .
$$

Thus at time $t=T_{0}$ we start again with a better estimate

$$
-M+(m-\varepsilon) \leq\left.(u(x, t)-\varphi(x)-c t)\right|_{t=T_{0}} \quad \text { for } x \in \mathbb{R}
$$

If $-2 \varepsilon \leq-M+(m-\varepsilon)$, then we use (3.14) and this estimate and obtain a further estimate.

$$
-M+2(m-\varepsilon) \leq\left.(u(x, t)-\varphi(x)-c t)\right|_{t=2 T_{0}} \quad \text { for } x \in \mathbb{R}, t \geq 2 T_{0} .
$$

We can repeat this argument until $\inf _{|x| \leq R}(u(x, t)-\varphi(x)-c t)$ becomes larger than $-2 \varepsilon$. Namely, we have

$$
\min \{-M+J(m-\varepsilon),-2 \varepsilon\} \leq\left.(u(x, t)-\varphi(x)-c t)\right|_{t=J T_{0}}
$$

for $x \in \mathbb{R}$ and any positive integer $J$. Here we can take $J$ as large as possible, that is,

$$
J=\left[\frac{M-2 \varepsilon}{m-\varepsilon}\right]+1
$$

and thus we have

$$
J \geq \frac{M-2 \varepsilon}{m-\varepsilon}
$$

Then we obtain

$$
-2 \varepsilon \leq\left.(u(x, t)-\varphi(x)-c t)\right|_{t=J T_{0}} \quad \text { for } x \in \mathbb{R} .
$$

and thus

$$
\varphi(x)+c t-2 \varepsilon \leq u(x, t) \quad \text { for all } x \in \mathbb{R}
$$

for sufficiently large $t>0$. This lower estimate combined with the upper estimate (3.2) completes the proof of Theorem 1.1. 
4. An example for non-asymptotically stability in $B C^{1}$. In this section, we always assume $\psi(x)$ belongs to $B C^{1}$, and does not decay as $|x| \rightarrow+\infty$. Fix $\varphi(x ; c)$ in Proposition 1.1 and we denote it simply by $\varphi(x)$. We will show that an example where $u(x, t)$ does not converges to $\varphi(x)+c t+\alpha$ for any fixed $\alpha \in \mathbb{R}$ for suitable choice of $\psi(x)$.

Let $\varepsilon_{1}>0$ be any given small number. Take $R_{5}>0$ so large that

$$
\left|\varphi(x)-\left(\frac{\sqrt{c^{2}-k^{2}}}{k} x+b\right)\right|<\varepsilon_{1} \quad \text { for }|x| \geq R_{5}
$$

holds true. Fix

$$
\Theta \in\left(0, \frac{1}{2} \min \left\{\theta_{0}, \frac{\pi}{2}-\theta_{0}\right\}\right)
$$

arbitrarily. Let $B\left(\left(x_{0}, y_{0}\right), r\right)$ be a ball with center $\left(x_{0}, y_{0}\right)$ and radius $r$. We will choose a sequence of positive integers $\{n(j)\}_{j=1}^{\infty}$ with

$$
\max \left\{\frac{1}{k}, R_{5}, 3 \varepsilon_{1}\right\}<n(1)<n(2)<\cdots<n(j)<\cdots \rightarrow+\infty
$$

as follows. Let $\left\{D_{j}(0)\right\}_{j=1}^{\infty}$ be defined by

$$
\begin{aligned}
D_{2 j-1}(0) & =B((x(2 j-1), y(2 j-1)), n(2 j-1)), \\
D_{2 j}(0) & =\overline{B((x(2 j), y(2 j), n(2 j)))} .
\end{aligned}
$$

We put $\Gamma_{j}(0)=\partial D_{j}(0)$, and choose $\{n(j)\}$ so that we have

(i) $\Gamma_{j}(0) \subset\{(x, y) \mid x>0, y>0\}, \quad \Gamma_{2 j-1}(0) \cap \Gamma_{2 j}(0)=\emptyset \quad$ for all $j \in \mathbb{N}$,

(ii) $\Gamma_{2 j-1}(0) \cap\left\{y=k^{-1} \sqrt{c^{2}-k^{2}} x+b+3 \varepsilon_{1}\right\}$ consists of one point, and $\Gamma_{2 j}(0) \cap$ $\left\{y=k^{-1} \sqrt{c^{2}-k^{2}} x+b-3 \varepsilon_{1}\right\}$ also consists of one point.

(iii) The center $(x(j), y(j))$ satisfies

$$
\begin{aligned}
x(2 j-1) & =\frac{\left(n(2 j-1)-3 \varepsilon_{1}\right) \cos \left(\theta_{0}-\Theta\right)}{\sin \Theta}, \\
y(2 j-1) & =\frac{\left(n(2 j-1)-3 \varepsilon_{1}\right) \sin \left(\theta_{0}-\Theta\right)}{\sin \Theta}+b, \\
x(2 j) & =\frac{\left(n(2 j)-3 \varepsilon_{1}\right) \cos \left(\theta_{0}+\Theta\right)}{\sin \Theta}, \\
y(2 j) & =\frac{\left(n(2 j)-3 \varepsilon_{1}\right) \sin \left(\theta_{0}+\Theta\right)}{\sin \Theta}+b
\end{aligned}
$$

for $j \in \mathbb{N}$.

For $x>R_{5}$, we take $\mathrm{D}(0)$ with

$$
\overline{D_{2 j-1}(0)} \subset \overline{D(0)} \subset \overline{D_{2 j}(0)} \quad \text { for all } j \in \mathbb{N}
$$

and

$$
\sup _{x \in(-\infty, \infty)}|u(x, 0)-\varphi(x)| \leq 4 \varepsilon_{1} .
$$

For $x \leq R_{5}$, we take $D(0)=\{y<\varphi(x)\}$. 


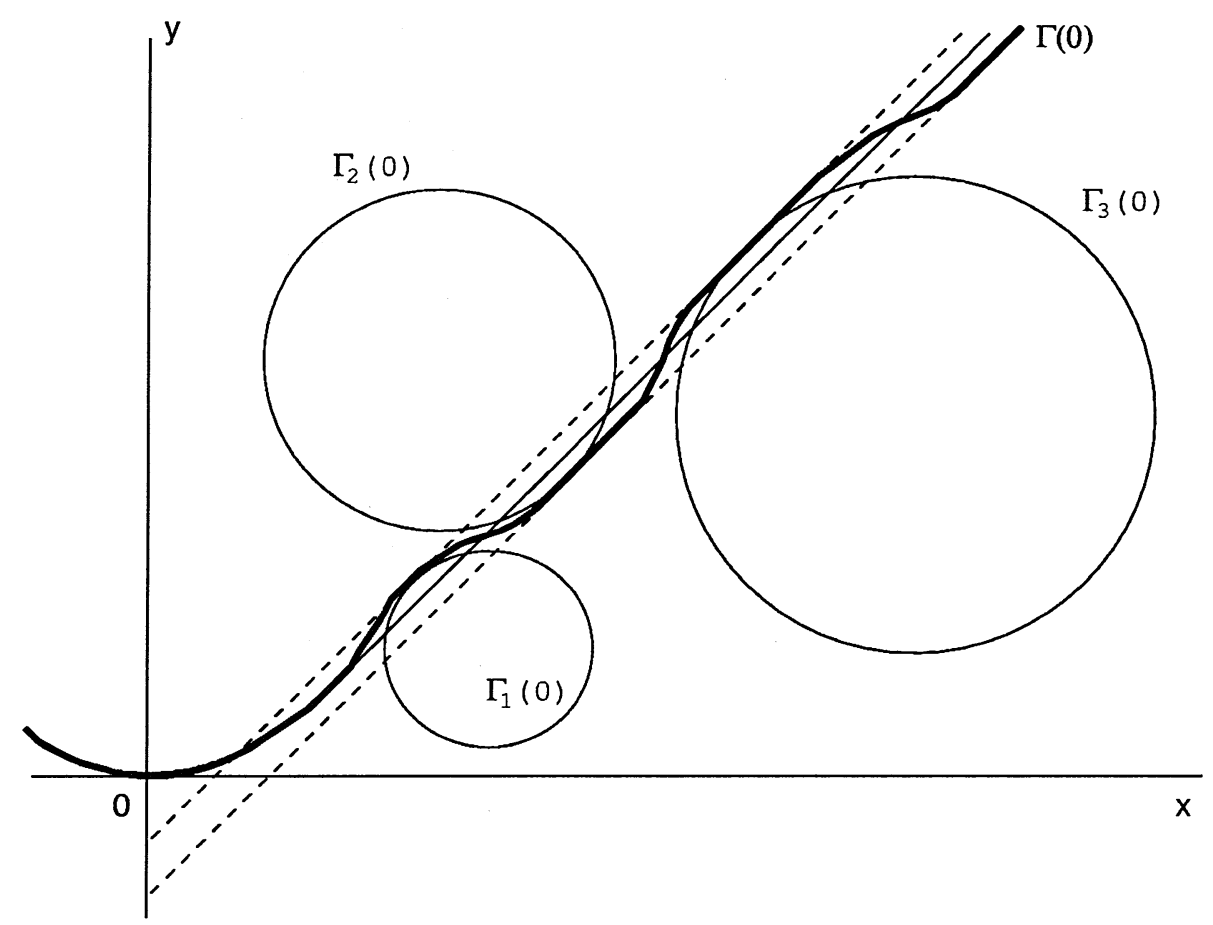

FIG. 4.1. A suitable choice of $\Gamma(0)$ for non-asymptotical stability

Then we have the following example for non-asymptotic stability of a traveling curved front $y=\varphi(x)+c t$.

THEOREM 4.1 Let $\varepsilon_{1}>0$ be arbitrarily given. There exists $\Gamma(t)$ which satisfies

$$
\sup _{x \in(-\infty, \infty)}|u(x, 0)-\varphi(x)| \leq 4 \varepsilon_{1}
$$

and

$$
\begin{gathered}
\Gamma(t) \cap\left\{y \geq \varphi(x)+\varepsilon_{1}+c t\right\} \neq \emptyset, \\
\Gamma(t) \cap\left\{y \leq \varphi(x)-\varepsilon_{1}+c t\right\} \neq \emptyset
\end{gathered}
$$

for all $t>0$. That is, $\Gamma(t)$ does not converges to $y=\varphi(x)+\alpha+$ ct in $L^{\infty}(\mathbb{R})$ for any fixed $\alpha \in \mathbb{R}$ (see Fig. 4.1).

Let $D_{j}(t)$ be the solution of (1.1) with an initial state $D_{j}(0)$. To prove Theorem 4.1, the following comparison principle is useful.

Lemma 4.1 For all $t \geq 0$ and all $j \in \mathbb{N}$, the following inclusion

$$
\overline{D_{2 j-1}(t)} \subset \overline{D(t)} \subset \overline{D_{2 j}(t)}
$$

follows from

$$
\overline{D_{2 j-1}(0)} \subset \overline{D(0)} \subset \overline{D_{2 j}(0)} .
$$


We will present the proof of this lemma later. First we prove Theorem 4.1 using this lemma.

Proof of Theorem 4.1. Let $r_{j}(t)$ be the radius of $\partial D_{j}(t)$. Then it satisfies

$$
\begin{aligned}
\frac{d r_{2 j-1}}{d t} & =-\frac{1}{r_{2 j-1}}+k \quad t>0, \\
r_{2 j-1}(0) & =n(2 j-1) .
\end{aligned}
$$

We compare $r_{2 j-1}(t)$ with $s_{2 j-1}(t)$ defined by

$$
\begin{gathered}
\frac{d s_{2 j-1}}{d t}=k \quad t>0, \\
s_{2 j-1}(0)=n(2 j-1)-3 \varepsilon_{1} .
\end{gathered}
$$

Then the distance between $\partial D_{2 j-1}(t)$ and $y=k^{-1} \sqrt{c^{2}-k^{2}} x+b$ is larger than or equals $r_{2 j-1}(t)-s_{2 j-1}(t)$. Let $T_{2 j-1}$ be the time with $r_{2 j-1}\left(T_{2 j-1}\right)-s_{2 j-1}\left(T_{2 j-1}\right)=$ $2 \varepsilon_{1}$. If $t \leq T_{2 j-1}$, then $\overline{D_{2 j-1}(t)} \cap\left\{y \geq k^{-1} \sqrt{c^{2}-k^{2}} x+b+2 \varepsilon_{1}+c t\right\} \neq \emptyset$ follows. By Lemma 4.1, $\Gamma(t) \cap\left\{y \geq k^{-1} \sqrt{c^{2}-k^{2}} x+b+2 \varepsilon_{1}+c t\right\} \neq \emptyset$ also follows if $t \leq T_{2 j-1}$. We estimate $T_{2 j-1}$ as follows. Integrating

$$
\frac{d}{d t}\left(r_{2 j-1}-s_{2 j-1}\right)=-\frac{1}{r_{2 j-1}}
$$

over $\left(0, T_{2 j-1}\right)$, we get

$$
\varepsilon_{1}=\int_{0}^{T_{2 j-1}} \frac{1}{r_{2 j-1}(t)} d t .
$$

Because $n(2 j-1)<r_{2 j-1}(t)$ for $t>0$, we have

$$
\varepsilon_{1}=\int_{0}^{T_{2 j-1}} \frac{1}{r_{2 j-1}(t)} d t<\frac{T_{2 j-1}}{n(2 j-1)}
$$

and hence

$$
T_{2 j-1}>\varepsilon_{1} n(2 j-1) \quad \text { for } j \in \mathbb{N} \text {. }
$$

Summing up the argument stated above, we have

$$
\Gamma(t) \cap\left\{y \geq \frac{\sqrt{c^{2}-k^{2}}}{k} x+b+2 \varepsilon_{1}+c t\right\} \neq \emptyset \quad \text { if } t<T_{2 j-1}
$$

for all $j \in \mathbb{N}$. From (4.1), we have

$$
\Gamma(t) \cap\left\{y \geq \varphi(x)+\varepsilon_{1}+c t\right\} \neq \emptyset
$$

requires at least $t>\varepsilon_{1} n(2 j-1)$ for all $j \in \mathbb{N}$.

Next, $r_{2 j}(t)$ satisfies

$$
\begin{aligned}
\frac{d r_{2 j}}{d t} & =-\frac{1}{r_{2 j}}-k \quad t>0 \\
r_{2 j}(0) & =n(2 j) .
\end{aligned}
$$


We consider $s_{2 j}(t)$ with

$$
\begin{gathered}
\frac{d s_{2 j}}{d t}=-k \quad t>0 \\
s_{2 j}(0)=n(2 j)-3 \varepsilon_{1} .
\end{gathered}
$$

Then the distance between $\partial D_{2 j}(t)$ and $y=\frac{\sqrt{c^{2}-k^{2}}}{k} x+b$ is larger than or equals $r_{2 j}(t)-s_{2 j}(t)$. Define $T_{2 j}$ by $r_{2 j}\left(T_{2 j}\right)-s_{2 j}\left(T_{2 j}\right) \stackrel{k}{=} 2 \varepsilon_{1}$. We estimate $T_{2 j}$ as follows. Integrating

$$
\frac{d}{d t}\left(r_{2 j}-s_{2 j}\right)=-\frac{1}{r_{2 j}} \quad t>0
$$

over $\left(0, T_{2 j}\right)$, we have

$$
\varepsilon_{1}=\int_{0}^{T_{2 j}} \frac{1}{r_{2 j}} d t
$$

For $0<t<T_{2 j}$, we have $s_{2 j}(t)<r_{2 j}(t)$. Using this inequality, we obtain

$$
\varepsilon_{1}=\int_{0}^{T_{2 j}} \frac{1}{r_{2 j}(t)} d t<\int_{0}^{T_{2 j}} \frac{1}{s_{2 j}(t)} d t
$$

Using $s_{2 j}(t)=n(2 j)-3 \varepsilon_{1}-k t$, we have

$$
T_{2 j}>\frac{\left(n(2 j)-3 \varepsilon_{1}\right)\left(1-e^{-k \varepsilon_{1}}\right)}{k} .
$$

If $t<T_{2 j}$, then $\partial D_{2 j}(t) \cap\left\{y \leq k^{-1} \sqrt{c^{2}-k^{2}} x+b-2 \varepsilon_{1}+c t\right\} \neq \emptyset$. Using (4.1), we obtain

$$
\Gamma(t) \cap\left\{y \leq \varphi(x)-\varepsilon_{1}+c t\right\} \neq \emptyset \quad \text { if } t<T_{2 j} .
$$

Thus $\Gamma(t) \cap\left\{y \leq \varphi(x)-\varepsilon_{1}+c t\right\} \neq \emptyset$ requires at least $t>k^{-1}\left(n(2 j)-3 \varepsilon_{1}\left(1-e^{-k \varepsilon_{1}}\right)\right)$ for some $j \in \mathbb{N}$.

Combining the arguments stated above, we obtain Theorem 4.1.

Now we state the proof of Lemma 4.1.

Proof of Lemma 4.1. We only show $\overline{D_{2 j-1}(t)} \subset \overline{D(t)}$. The other inequality can be proved similarly.

Assume the contrary. Then there exists $T>0$ such that $\overline{D_{2 j-1}(T)} \not \subset \overline{D(T)}$. Let $\left(x_{0}(T), y_{0}(T)\right)$ be any one with $\left(x_{0}(T), y_{0}(T)\right) \in \partial D_{2 j-1}(T)$ and $\left(x_{0}(T), y_{0}(T)\right) \notin$ $\overline{D(T)}$.

Define

$$
\tau=\inf \left\{T>0 \mid \text { there exists }\left(x_{0}(T), y_{0}(T)\right)\right\}
$$

Taking a subsequence, $\left(x_{0}(T), y_{0}(T)\right)$ converges to $\left(x_{\infty}, y_{\infty}\right)$ as $T \rightarrow \tau+0$. This is because $\left\{\partial D_{2 j-1}(T)\right\}_{\tau \leq T \leq \tau+1}$ is compact. We have $\left(x_{\infty}, y_{\infty}\right) \in \partial D_{2 j-1}(\tau) \cap \partial D(\tau)$. Recall that $\Gamma(t)=\partial D(t)$ and $D(t)=\{y<u(x, t)\}$. In some neighborhood of $\left(x_{\infty}, y_{\infty}\right), D_{2 j-1}(t)=\{y<w(x, t)\}$, where $w(x, t)$ is a solution of $(1.2)$. Then there 
exist $x_{1}$ and $x_{2}\left(x_{1}<x_{\infty}<x_{2}\right)$ with $w\left(x_{j}, \tau\right)<u\left(x_{j}, \tau\right)$ for $j=1,2$. This is because $\partial D_{2 j-1}(\tau)$ is a circle, while $u_{x}(x, t)$ satisfies

$$
\sup _{x \in(-\infty, \infty)}\left|u_{x}(x, \tau)\right|<+\infty
$$

from Theorem 1.4 of [9]. There exists $\tau^{\prime}>\tau$ with

$$
w\left(x_{j}, t\right)<u\left(x_{j}, t\right) \quad \text { for } t \in\left[\tau, \tau^{\prime}\right), j=1,2
$$

From the definition of $\tau$, we have

$$
w(x, \tau) \leq u(x, \tau) \quad \text { for } x \in\left[x_{1}, x_{2}\right] .
$$

By the comparison principles for parabolic equations [10], we have

$$
w(x, t) \leq u(x, t) \quad \text { for } x \in\left[x_{1}, x_{2}\right], t \in\left[\tau, \tau^{\prime}\right) .
$$

This contradicts the definition of $\tau$. We complete the proof of Lemma 4.1.

Acknowledgements. The authors would like to express their gratitude to Professor Eiji Yanagida of Tohoku University for his comments on this paper. The first author is supported in part by Grant-in-Aid for Encouragement of Young Scientists (No. 11740077), The Ministry of Education, Science, Sports and Culture, and Ryukoku University Science and Technology Fund. This work is done while the second author is visiting Georgia Institute of Technology as a dispatched researcher of the same ministry of Japan.

\section{REFERENCES}

[1] P. K. BRAZHNIK, Exact solutions for the kinematic model of autowaves in two-dimensional excitable media, Physica D, 94 (1996), pp. 205-220.

[2] P. K. BRAZHNiK AND V. A. Davydov, Non-spiral autowave structures in unrestricted excitable media, Physics Letters, A 199 (1995), pp. 40-44.

[3] X. CHEN, Generation and propagation of interfaces for reaction-diffusion equations, J. Diff. Equation, 96 (1992), pp. 116-141

[4] K.-S. CHOU AND Y.-C. KWONG, On quasilinear parabolic equations which admit global solutions for initial data with unrestricted growth, Calc. Var. Partial Differential Equations, 12, no. 3 (2001), pp. 281-315

[5] K. Deckelnick, C. M. Elliott and G. Richardson, Long time asymptotics for forced curvature flow with applications to the motion of a superconducting vortex, Nonlinearity, 10 (1997), pp. 655-678.

[6] P. C. FIFE, Dynamics of Internal Layers and Diffusive Interfaces, (CBMS-NSF Reg. Conf. Ser. Appl. Math. 53, 1988), SIAM, Philadelphia.

[7] O. A. Ladyženskaja, V. A. Solonnikov and N. N. Ural'Ceva, Linear and Quasilinear Equations of Parabolic Type, Translations of Mathematical Monographs 23, Providence RI, American Mathematical Society, 1968.

[8] V. Pérez-Muñuzuri, M. Gómez-Gesteira, A. P. Muñuzuri, V. A. Davydov and V. PérezVILlaR, V-shaped stable nonspiral patterns, Physical Review E, 51 (1995), pp. 845-847.

[9] H. NinomiYa AND M. TANiguchi, Traveling curved fronts of a mean curvature flow with constant driving force. Free Boundary Problems: Theory and Applications I, Mathematical Sciences and Applications, 13, Gakuto International Series, (2000) pp. 206-221, Gakkotosho, Tokyo.

[10] M. H. Protter And H. F. Weinberger, Maximum Principles in Differential Equations, Prentice-Hall Inc., 1967.

[11] H. YAGISITA, Nearly spherically symmetric expanding fronts in a bistable reaction-diffusion equation, J. Dynam. Differential Equations, 13 no. 2 (2001), pp. 323-353. 
H. NINOMIYA AND M. TANIGUCHI 